\title{
Acrokeratosis verruciformis of Hopf - Case report ${ }^{*}$
}

\author{
Tatiana Cristina Pedro Cordeiro de Andrade ${ }^{1}$ \\ Tatiane Meira Pinho Silva ${ }^{1}$ \\ Adauto José Ferreira Nunes ${ }^{1}$
}

\author{
Gardênia Viana da Silva ${ }^{1}$ \\ Ana Cecília Versiani Duarte Pinto ${ }^{1}$ \\ Antônio Carlos Ceribelli Martelli ${ }^{1}$
}

DOI: http:/ /dx.doi.org/10.1590/abd1806-4841.20164919

\begin{abstract}
A 54 year-old woman with a 3-year history of rheumatoid arthritis (RA) consulted us because of weight loss, fever and skin eruption. On physical examination, erythematous plaques with a pseudo-vesicular appearance were seen on the back of both shoulders. Histological examination was consistent with rheumatoid neutrophilic dermatosis (RND). After three days of prednisone treatment, the skin eruption resolved. RND is a rare cutaneous manifestation of seropositive RA, characterized by asymptomatic, symmetrical erythematous plaques with a pseudo-vesicular appearance. Histology characteristically reveals a dense, neutrophilic infiltrate with leucocitoclasis but without other signs of vasculitis. Lesions may resolve spontaneously or with RA treatment. This case illustrates an uncommon skin manifestation of active rheumatoid arthritis.
\end{abstract}

Keywords: Arthritis; Arthritis, rheumatoid; Rheumatoid factor

\section{INTRODUCTION}

Acrokeratosis verruciformis of Hopf $(\mathrm{AVH})$ is a rare genodermatosis characterized by keratotic lesions on the dorsum of the hands and feet. ${ }^{1-6}$ Lesions are usually present from birth or early childhood, but some cases in adult life are reported. ${ }^{1,4,7,8}$ Differential diagnosis includes epidermodysplasia verruciformis, stucco keratosis, and Darier's disease. We report a patient with exuberant clinical lesions and highlight the importance of clinical and histopathological examination for differential diagnosis.

\section{CASE REPORT}

We report a 58-year-old white male patient presented with multiple warts on the hands and feet. Lesions started at the age of eight years with progressive growth. Personal history revealed unspecified arrhythmia, but patient did not use any medication. Family history revealed that the father of the patient had similar lesions. Dermatological examination showed multiple brownish flat-topped warty plaques on the hands and feet and nail dystrophy (Figures 1 and 2). Biopsy of the lesions revealed irregular epithelial hyper-
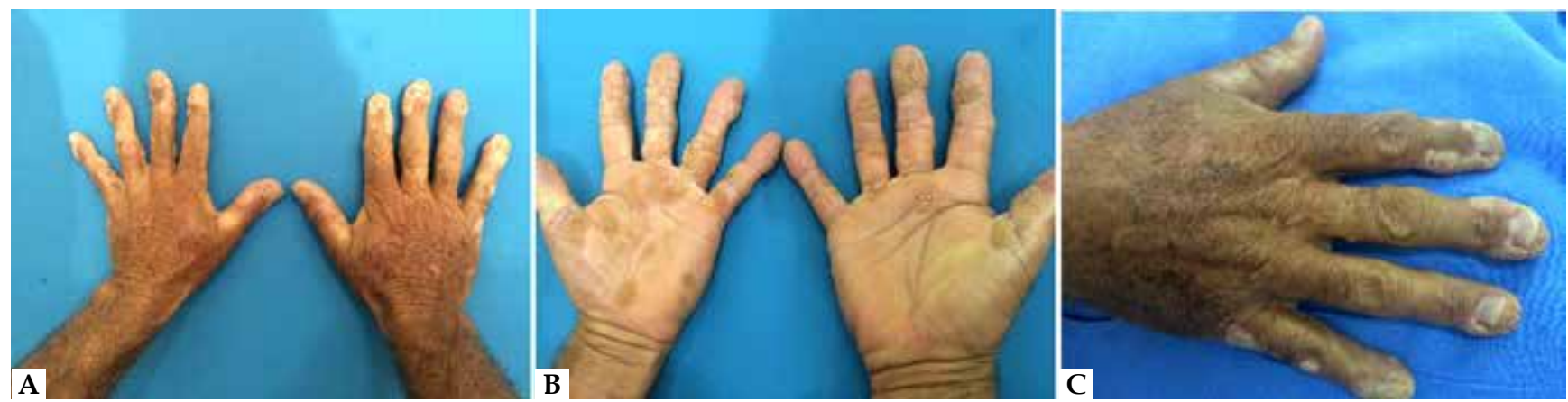

FIGURE 1: Multiple flat-topped brownish verrucous plaques on the dorsum and palms of the hands associated with nail dystrophy 
plasia, hyperkeratosis and papillomatosis, histologically compatible with acrokeratosis verruciformis of Hopf (Figure 3). Our therapeutic attempt with salicylic acid topical 3\% showed no clinical improvement. Then, we prescribed acitretin $25 \mathrm{mg}$ every other day with satisfactory results. The patient is being followed up.

\section{DISCUSSION}

Acrokeratosis verruciformis of Hopf (AVH) was first described by Hopf in 1931. ${ }^{1,4,7-9}$ The disease follows a chronic course without spontaneous remission. It has an autosomal dominant pattern of inheritance with incomplete penetrance, which may explain the difficulty of finding similar cases in the family. Sporadic cases with later onset have also been reported..$^{1,3,5,7-9}$ The disease has no gender preference. ${ }^{1,78}$ Its probable etiology would be a mutation in ATP2A2 gene located on chromosome 12q24. This heterozygous pro602Leu mutation (P602L) in ATP2A2 leads to amino acid substitutions within the ATP binding domain. ${ }^{1-3,9}$

Some authors describe AVH as a variant of Darier-White's disease due to clinical similarities and inheritance pattern. However, Darier's disease features dyskeratotic cells, has a predilection for sebaceous areas, can affect the oral mucosa, and presents no signs of carcinomatous transformation. ${ }^{1-4,7,8,9}$ Dhitavat et al. studied a family affected with AVH in six generations and analyzed a P602L mutation in ATP2A2. The study showed that P602L lost its ability to transport calcium due to the loss of function of the sarcoendoplasmic reticulum Ca2+ ATPase2, similar to Darier's disease. The similarities provide evidence that acrokeratosis verruciformis and Darier's disease are allelic disorders. ${ }^{9}$

Acrokeratosis clinically manifests itself as flat-topped, polygonal, papules and verrucous plaques. The brownish to skin-colored lesions have hard consistency, and its friction can produce vesicles. ${ }^{1-5,7,9}$ The lesions are usually located on the back of the proximal and distal interphalangeal joints of the hands and feet. ${ }^{1,4}$ Less frequently, lesions occur on the legs, knees, arms, and elbows - as presented by our patient - and rarely on other parts of body. ${ }^{1,4,7}$ The disease does not affect sebaceous areas - frontal scalp, flexures, or oral mucosa. ${ }^{7}$ The disease also features punctate keratosis on the palms and soles. Nail changes are frequently reported, such as thickening of the nail plate, leukonychia and longitudinal ridges and nicks in the free edges. ${ }^{1,4,7,8}$

Previously reported cases by Ormond et al. and Santos et al. presented with less exuberant clinical lesions demonstrate the wide clinical spectrum of verruciformis acrokeratosis of Hopf. ${ }^{78}$

Diagnosis is defined by histopathological features that include papillomatosis (circumscribed epidermal elevations known as "church spires), acanthosis, hyperkeratosis, and hypergranulosis without parakeratosis. ${ }^{14,7,8,9}$ Although the hypertrophic variant of seborrheic keratosis may present similar histological changes,
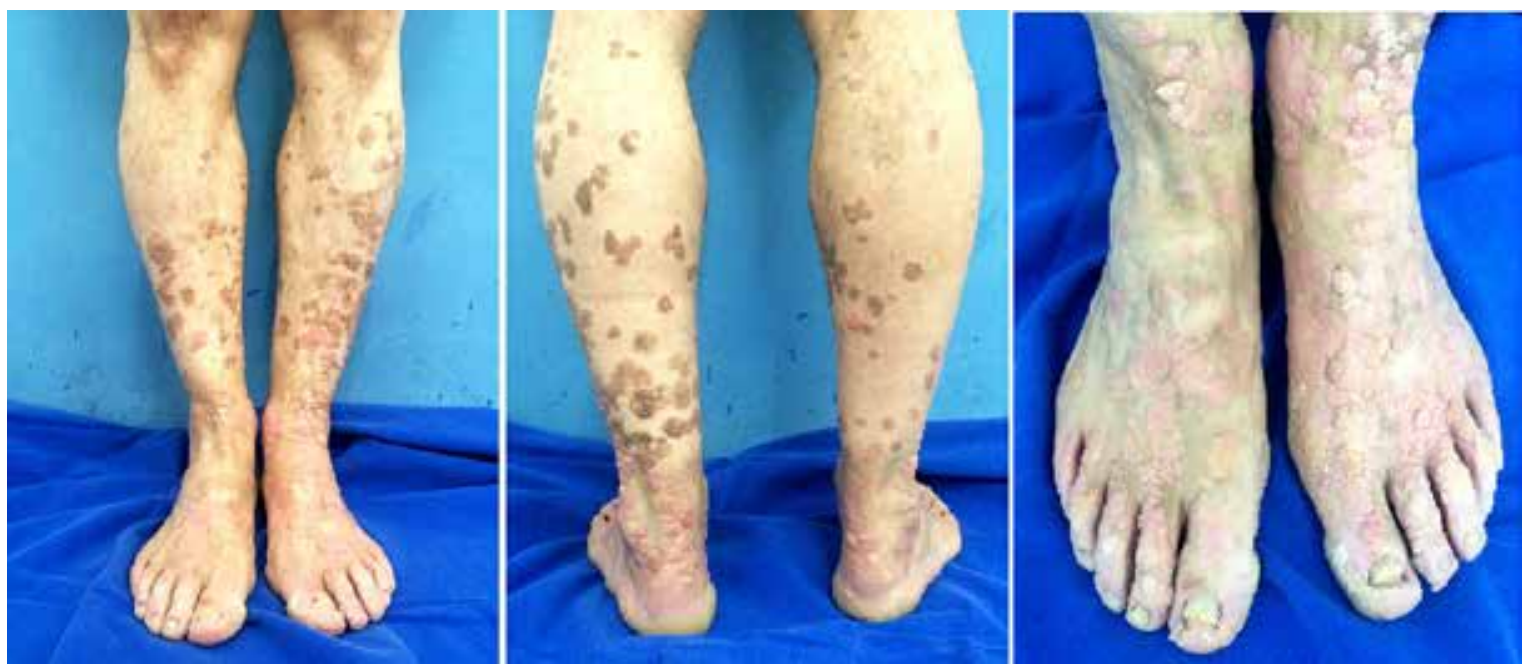

Figure 2:

Multiple flattopped brownish verrucous plaques on the dorsum of the feet and distal lower limbs associated with nail dystrophy

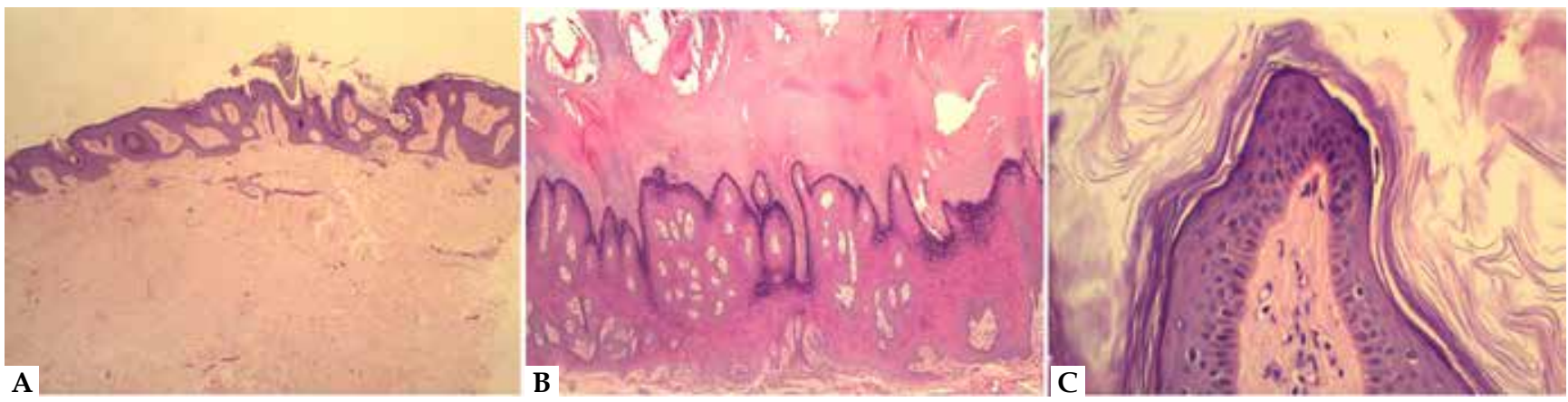

FIgURE 3: Histology. A - HE 20X: epithelial hyperplasia, hyperkeratosis and papillomatosis. B - HE 20X: epithelial hyperplasia, exuberant hyperkeratosis and papillomatosis. C - HE 200X: in detail, dermal papilla with hyperkeratosis presenting a church-spire pattern. 
clinical findings are completely different. ${ }^{1}$ Squamous cell carcinoma transformation may occur although it is rare phenomenon. ${ }^{6,7}$

The only effective treatment available is superficial ablation. ${ }^{4}$ Although not the recommended treatment due to frequent recurrences, cryotherapy, laser therapy and surgical excision are other options. ${ }^{4}$ Conservatively, keratolytic solutions can be applied. Some reports describe the positive effects of acitretin on the lesions - as observed in our patient - and on the reduction of malignant transformations. ${ }^{5,6}$
Ormond et al. and Santos et al. opted for treatment with topical keratolytics. In the present case, we observed no improvement with the use of that medication. ${ }^{7,8}$

We report a case of $\mathrm{AVH}$ with exuberant clinical lesions and highlight the importance of clinical and histopathological examination for differential diagnosis that includes Darier's disease, epidermodysplasia verruciformis, flat warts, lichen planus and lichen nitidus. ${ }^{1,2,4,7,8}$

\section{REFERENCES}

1. Bang CH, Kim HS, Park YM, Kim HO, Lee JY. Non-familial acrokeratosis verruciformis of Hopf. Ann Dermatol. 2011;23:S61-3.

2. Bergman R, Sezin T, Indelman M, Helou WA, Avitan-Hersh E. Acrokeratosis verruciformis of Hopf showing P602L mutation in ATP2A2 and overlapping histopathological features with Darier disease. Am J Dermatopathol. 2012;34:597601.

3. Wang PG, Gao M, Lin GS, Yang S, Lin D, Liang YH, et al. Genetic heterogeneity in acrokeratosis verruciformis of Hopf. Clin Exp Dermatol. 2006;31:558-63.

4. Nair PA. Acrokeratosis verruciformis of hopf along lines of Blaschko. Indian J Dermatol. 2013;58:406.

5. Serarslan G, Balci DD, Homan S. Acitretin treatment in acrokeratosis verruciformis of Hopf. J Dermatolog Treat. 2007;18:123-5.

6. Farro P, Zalaudek I, Ferrara G, Fulgione E, Cicale L, Petrillo G, et al. Unusual association between acrokeratosis verruciformis of Hopf and multiple keratoacanthomas. Successful therapy with acitretin. J Dtsch Dermatol Ges. 2004;:2:440-2.

7. Ormond DTS, Viana SS, Vitral EAO, Pereira CAC, Carvalho MTF. Acroceratose verruciforme de Hopf: relato de caso. An Bras Dermatol 1998;73:25-7.

8. Santos OLR, Cardoso ICL, Cardoso AMF, Filho RS. Acroceratose verruciforme de Hopf. An Bras Dermatol 1995;70:17-9.

9. Dhitavat J, Macfarlane S, Dode L, Leslie N, Sakuntabhai A, MacSween R, Saihan $\mathrm{E}$, et al. Acrokeratosis verruciformis of Hopf is caused by mutation in ATP2A2: evidence that it is allelic to Darier's disease. J Invest Dermatol. 2003;120:229-32.

MAILING ADDRESS:

Tatiana Cristina Pedro Cordeiro de Andrade

R. Dr. Plínio Barreto, 173 - Apto 21A

Condomínio Vista Bela

Bairro Bela Vista

01313-020 - São Paulo - SP

Brazil

E-mail: tatianap.andrade@gmail.com

How to cite this article: Andrade TCPC, Silva GV, Silva TMP, Pinto ACVD, Nunes AJF, Martelli ACC. Acrokeratosis verruciformis of Hopf - Case report. An Bras Dermatol. 2016;91(5):639-41. 